\title{
Thermal conductivity of GaAs nanowires studied by micro-Raman spectroscopy combined with laser heating
}

\author{
Martin Soini, ${ }^{1}$ Ilaria Zardo, ${ }^{1}$ Emanuele Uccelli, ${ }^{1,2}$ Stefan Funk, ${ }^{1}$ Gregor \\ Koblmüller, ${ }^{1}$ Anna Fontcuberta i Morral, ${ }^{1,2}$ and Gerhard Abstreiter ${ }^{1}$ \\ ${ }^{1}$ Walter Schottky Institut and Physik Department, \\ Technische Universität München, Am Coulombwall 3, D-85748 Garching, Germany \\ ${ }^{2}$ Laboratoire des Matériaux Semiconducteurs, Institut des Matériaux, \\ Ecole Polytechnique Fédérale de Lausanne, CH-1015 Lausanne, Switzerland
}

\begin{abstract}
The thermal properties of freely suspended GaAs nanowires are investigated by applying a method which relies on laser heating and the determination of the local temperature by Raman spectroscopy. In order to determine the values for the thermal conductivity $\kappa$, the fraction of the laser power absorbed inside the GaAs nanowire is estimated by numerical simulations. The thermal conductivity of nanowires with homogeneous diameter is found to lie in the range of $8-36 \mathrm{Wm}^{-1} \mathrm{~K}^{-1}$. The change of the temperature profile in the presence of a tapering was investigated. Furthermore, we discuss the influence of laser heating in ambient conditions on the value of $\kappa$.
\end{abstract}

The role of thermoelectrics as a competitive technology is, up to now, inhibited by the interdependences of the relevant material parameters, which determine the thermoelectric figure of merit ZT. Recently, the search for materials with high electrical and low thermal conductivity has increased the interest in nanoscale systems for thermoelectric application [1]. Nanowires (NWs) are promising candidates for the reduction of the thermal conductivity due to increased boundary scattering, while keeping the electrical conductivity relatively high [2-6].

In this work we investigate the thermal conductivity $\kappa$ of GaAs NWs. Our method is based on the laser heating of freely suspended NWs and the determination of the local temperature by micro-Raman spectroscopy. The conductivity is found to be significantly lower in NWs than in bulk GaAs. Furthermore, our results confirm recent theoretical calculations by Martin et al. [7].

We use zincblende NWs grown by gallium-assisted molecular beam epitaxy, exhibiting very high structural quality [8] and diameters $d$ between 150 and $170 \mathrm{~nm}$. The facets belong to the $\{110\}$ family and the NW axis corresponds to the [111] direction. The typical Raman spectra obtained from GaAs NWs mainly show the GaAs transverse optical (TO) peak at $268.7 \mathrm{~cm}^{-1}(300 \mathrm{~K})$ and a weak peak of the strongly attenuated forbidden longitudinal optical (LO) mode at $292.2 \mathrm{~cm}^{-1}[8,9]$. The TO peak is of only interest for our work, since the shift of its position provides information about the local temperature. Details about the NW growth and the corresponding properties can be found elsewhere [8, 10-12].

The NWs are transferred by dropcasting to a silicon substrate containing large arrays of irregularly shaped gold pads of $\approx 3 \mu \mathrm{m}$ radius. The pads are $300 \mathrm{~nm}$ high and are fabricated by e-beam evaporation after a standard photolithographic step. For the measurements we choose NWs which are freely suspended between two pads on a length of $2-4 \mu \mathrm{m}$ (see FIG. 1a). The Raman spectra are recorded in backscattering geometry with an XY Dilor triple spectrometer equipped with a multichannel CCD. The $514.5 \mathrm{~nm}$ line of an $\mathrm{Ar}^{+}$laser is used for lo-

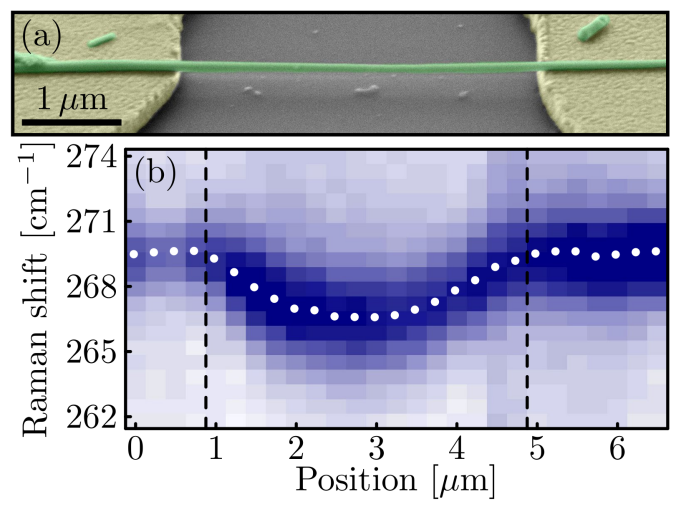

FIG. 1. (a) (Color online) Scanning electron micrograph of the typical sample configuration: The NW is freely suspended between two gold pads. (b) Spatially resolved Raman measurement on a single NW. The maxima of the TO peaks are highlighted by white dots. The dashed lines visualize the gold pad edges. Darker colors correspond to higher intensity [a.u.].

cal heating of the NWs and as excitation for Raman spectroscopy simultaneously. The beam is focused through a $100 \times$, N.A. $=0.95$ microscope objective for measurements in air and a $63 \times$, N.A. $=0.75$ objective for measurements in vacuum $\left(10^{-5}\right.$ mbar $)$ in a vacuum chamber through a $1.5 \mathrm{~mm}$ thick glass window. The corresponding Gaussian shaped spot shows a FWHM of $540 \mathrm{~nm}$ and $720 \mathrm{~nm}$, respectively. The samples are positioned on a piezo stage to scan along the NW with a step size of $0.25-1 \mu \mathrm{m}$. The polarization of the excitation light is set parallel to the NW axis in order to maximize the absorption and thus the heating effect as well as the Raman scattering intensity [8]. Our method is based on the determination of the local temperature of the NW while heating it with constant laser power at several discrete spots. The local temperature is calculated from the linear relation between temperature and the GaAs TO Raman peak shift, which is given by $\mathrm{d} \nu / \mathrm{d} T=0.016 \mathrm{~K}^{-1} \mathrm{~cm}^{-1}$ for bulk [13]. It has been shown, that this dependence 
is the same for bulk and NWs with $d$ as small as $33 \mathrm{~nm}$ for $\mathrm{Si}$ [14]. In FIG. 1b we give an example of the Raman spectra obtained during a scan along a NW. The position-dependent shift of the TO peak is clearly visible.

A simple model to describe the expected temperature profile on the suspended part of the NW is given by Hsu et al. [15], which predicts a parabola of the form

$$
\Delta T(x)=-\frac{P_{\mathrm{abs}}}{\kappa A}\left(x^{2}-L / 4\right)=-B_{1} x^{2}+\Delta T_{\max },
$$

with $P_{\text {abs }}$ the power absorbed inside the NW, $L$ the length of the suspended part, and $A$ the cross section area. The origin of the coordinate system is set to the center of the suspended part. We use the maximum temperature difference $\Delta T_{\max }$ obtained from the fit to the measured parabolic profile for the calculation of the thermal conductivity $\kappa=P_{\text {abs }} L /\left(4 \Delta T_{\max } A\right)$. The absorbed power is extracted from ab-initio finite differences simulations, performed with the software package MEEP [16].

We simulate the system containing a NW with hexagonal cross-section, the silicon substrate underneath, as well as a Gaussian shaped transverse magnetic (TM) laser source with a FWHM equal to the measured width of the focus in our setup. The absorption extracted from the simulations is defined as the fraction of the power dissipated inside the NW divided by the total power of the incoming beam. In FIG. 2 we present simulated diameter dependencies for different distances $z$ between the NW and the substrate. Several peaks are visible, which are attributed to specific resonances [17]. Furthermore, the strong influence of the substrate on the absorption is evident.

In table I we present examples of values of $\kappa$ obtained from the parabolic fits to the measured temperature profiles shown in FIG. 3. All these measurements were performed on the same NW $(d=160 \mathrm{~nm})$, by starting with those in vacuum. The obtained values for $\kappa$ are smaller than those reported for bulk GaAs $\left(54 \mathrm{Wm}^{-1} \mathrm{~K}^{-1}\right)$ [18]. Two trends can be observed. First of all, $\kappa$ gets smaller with increasing power and $\Delta T_{\max }$. In vacuum this can be attributed mainly to the temperature dependence of $\kappa$.

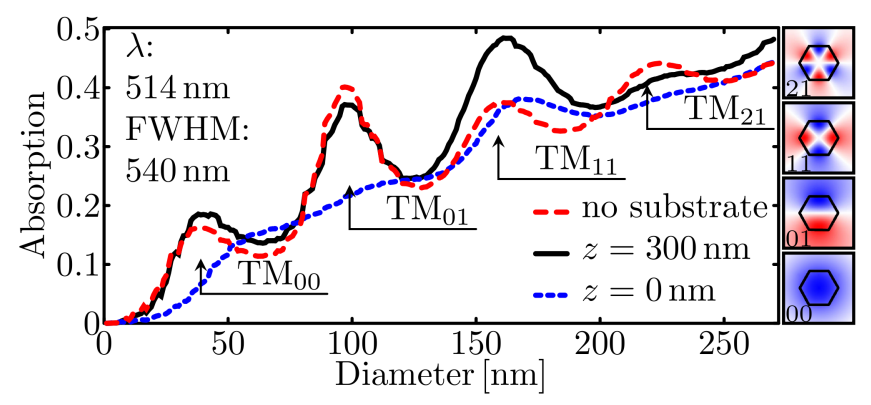

FIG. 2. Fraction of the incident laser power absorbed inside the NW for different substrate configurations. The peaks are attributed to the TM modes shown on the right hand side.

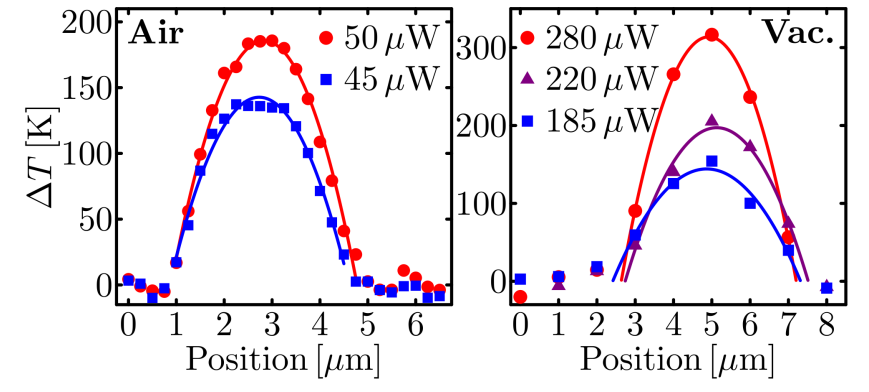

FIG. 3. Example of parabolic temperature profiles obtained for homogeneous NWs. Symbols indicate experimental data points, solid lines are parabolic fits. (diameter: $160 \mathrm{~nm}$ ).

\begin{tabular}{l|r|l|l|r} 
& $\boldsymbol{P}[\mu \mathrm{W}]$ & $\boldsymbol{T}_{\max }[\mathrm{K}]$ & $\boldsymbol{\kappa}\left[\mathrm{Wm}^{-1} \mathrm{~K}^{-1}\right]$ \\
\hline \hline \multirow{3}{*}{ Vacuum } & 185 & $130 \pm 12$ & $36 \pm 4$ \\
& 220 & $187 \pm 14$ & $29 \pm 3$ \\
& 280 & $314 \pm 4$ & $22 \pm 2$ \\
\hline \multirow{2}{*}{ Air } & 45 & $142 \pm 3$ & $9 \pm 1$ \\
& 50 & $187 \pm 2$ & $8 \pm 1$
\end{tabular}

TABLE I. Values of $\kappa$ calculated from the profiles in FIG. 3

In ambient air, irreversible changes are observed, probably due to oxidation of the wires at higher temperatures. Secondly, the values for $\kappa$ obtained in vacuum are notably higher than the ones obtained from the measurements in air. This might also be due to the oxidation and the formation of amorphous arsenic by laser induced heating, since the modes associated to the formation of arsenic as well as oxide can be observed for higher power conditions [19].

Recently, theoretical calculations of $\kappa$ for GaAs NWs have been published by Martin et al. [7]. For smooth GaAs NWs with $d$ between 100 and $200 \mathrm{~nm}$ and temperatures between 300 and $400 \mathrm{~K}$ they report values of $\kappa$ between 11 and $20 \mathrm{Wm}^{-1} \mathrm{~K}^{-1}$. Our measured values are in the range between 8 and $36 \mathrm{Wm}^{-1} \mathrm{~K}^{-1}$. In general, those of our values where the oxidation effects can be assumed to be the lowest ( $45 \mu \mathrm{W}$ in FIG. 4 and $185 \mu \mathrm{W}$ in table I), are higher than the theoretical calculations.

The power dependence of $\kappa$ is shown in FIG. 4. It was measured in air from low to high power at first, followed by the measurement at low power (about $46 \mu \mathrm{W}$ ), marked as red dot. We attribute the irreversible reduction of the thermal conductivity to the formation of an oxide shell around the NW because of laser annealing.

We also performed measurements on NWs with inhomogeneous diameter. The NW in FIG. 5a shows a tapering toward lower diameter near the left gold pad. The linear temperature profile indicates that this transition leads to an asymmetry in the thermal resistance. This follows from the model of $\mathrm{Hsu}$ et al. [15], where linear profiles are attributed to large asymmetric contact resistances. Therefore the temperature behavior in this example illustrates the decrease of the thermal conductance 


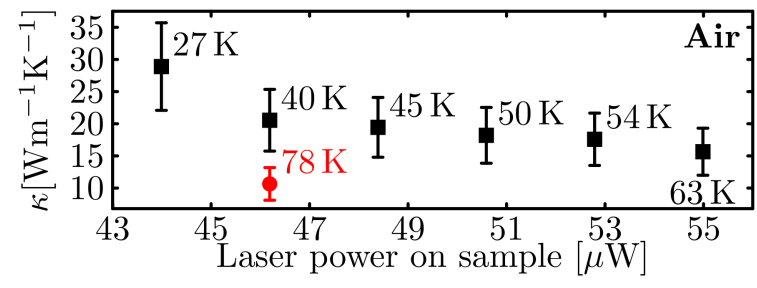

FIG. $4 . \kappa$ for a single $170 \mathrm{~nm}$ NW measured at different powers. The data point indicated by the red dot was measured at last. The temperatures indicate the $\Delta T_{\max }$. We observe irreversible changes with increasing laser power.

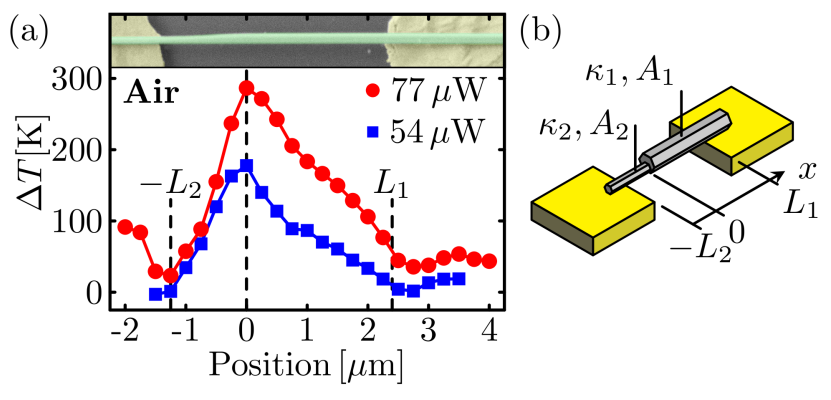

FIG. 5. (a) Measurement performed on a NW with inhomogeneous diameter. (b) Model used for the estimation of $\kappa_{1}$.

per length with decreasing NW diameter. We use the maximum temperatures in FIG. 5a to estimate the upper limit of $\kappa_{1}$ (see FIG. 5b). In the limit of infinite thermal resistance on the left side of the transition $\left(\kappa_{2}=0\right)$, the model yields $\kappa_{1}^{\max }=P_{\mathrm{abs}} L_{1} /\left(\Delta T_{\max } A_{1}\right)$. With the length $L_{1}=2.32 \mu \mathrm{m}$, the diameter $d_{1}=165 \mathrm{~nm}$, the simulated absorption 0.48 as well as $\Delta T_{\max }=250 \mathrm{~K}$ and $\Delta T_{\max }=180 \mathrm{~K}$ for the measurements at a power of $77 \mu \mathrm{W}$ and $54 \mu \mathrm{W}$ respectively, we obtain $\kappa_{1}^{\max }=$ $19 \pm 0.3 \mathrm{Wm}^{-1} \mathrm{~K}^{-1}$.

In summary, we present the determination of the thermal conductivity of GaAs NWs by laser heating and Raman spectroscopy. The influence of the measurement process in terms of laser annealing is apparent especially in ambient environment. Our results are in good agreement with theoretical predictions. We demonstrate the high consistency of the local temperature measurement based on micro-Raman spectroscopy by applying this method to tapered nanowires.

\section{ACKNOWLEDGMENTS}

We want to thank M. Bichler for excellent experimental help. This work was supported financially by the Marie Curie Excellence Grant "SENFED", the DFG via the excellence cluster Nanosystems Initiative Munich (NIM) and the Institute for Advanced Study of the TU München.
[1] G. J. Snyder, and E. S. Toberer, Nature Materials 7, 105 (2008).

[2] A. I. Hochbaum, R. Chen, R. D. Delgado, W. Liang, E. C. Garnett, M. Najarian, A. Majumdar, and P. Yang, Nature 451, 163 (2008).

[3] A. I. Boukai, Y. Bunimovich, J. Tahir-Kheli, J.-K. Yu, W. A. Goddard III, and J. R. Heath, Nature 451, 168 (2008).

[4] D. G. Cahill, W. K. Ford, K. E. Goodson, G. D. Mahan, A. Majumdar, H. J. Maris, R. Merlin, and S. R. Phillpot, J. App. Phys. 93, 793 (2003).

[5] D. Li, Y., P. Kim, L. Shi, P. Yang, and A. Majumdar, Appl. Phys. Lett. 83, 2934 (2003).

[6] A. L. Moore, S. K. Saha, R. S. Prasher, and L. Shi, Appl. Phys. Lett. 93, 083112 (2008).

[7] P. N. Martin, Z. Aksamija, E. Pop, and U. Ravaioli, Nano Lett. 10, 1120 (2010).

[8] I. Zardo, S. Conesa-Boj, F. Peiro, J. R. Morante, J. Arbiol, E. Uccelli, G. Abstreiter, and A. Fontcuberta i Morral, Phys. Rev. B 80, 245324 (2009).

[9] D. Spirkoska, G. Abstreiter, and A. Fontcuberta i Morral, Nanotechnology 19, 435704 (2008).

[10] D. Spirkoska, C. Colombo, M. Heiss, G. Abstreiter, and A. Fontcuberta i Morral, J. Phys.: Condens. Matter 20,
454225 (2008).

[11] C. Colombo, D. Spirkoska, M. Frimmer, G. Abstreiter, and A. Fontcuberta i Morral, Phys. Rev. B 77, 155326 (2008).

[12] A. Fontcuberta i Morral, C. Colombo, G. Abstreiter, J. Arbiol, and J. R. Morante, App. Phys. Lett. 92, 063112 (2008).

[13] J. M. Besson, J. P. Iti, A. Polian, G. Weill, J. L. Mansot, and J. Gonzalez, Phys. Rev. B 44, 4214 (1991).

[14] G. S. Doerk, C. Carraro, and R. Maboudian, Phys. Rev. B 80, 073306 (2009).

[15] I.-K. Hsu, S. B. Cronin, R. Kumar, and A. Bushmaker, App. Phys. Lett. 92, 063119 (2008).

[16] A. F. Oskooi, D. Roundy, M. Ibanescu, P. Bermel, J. D. Joannopoulos, and S. G. Johnson, Comp. Phys. Comm. 181, 687 (2010).

[17] L. Cao, J. S. White, J.-S. Park, J. A. Schuller, B. M. Clemens, and M. L. Brongersma, Nature Materials 8, 643 (2009).

[18] R. O. Carlson, G. A. Slack, and S. J. Silverman, J. Appl. Phys. 36, 505 (1965).

[19] I. H. Campbell, and P. M. Fauchet App. Phys. Lett. 57, 10 (1990). 